\title{
ON TOPOLOGICAL DEFECT FORMATION IN THE PROCESS OF SYMMETRY BREAKING PHASE TRANSITIONS
}

\author{
Eleonora Alfinito, Oreste Romei and Giuseppe Vitiello \\ Dipartimento di Fisica, INFN and INFM \\ Università di Salerno, 84100, Italia \\ e-mail: alfinito@sa.infn.it,romei@sa.infn.it, vitiello@sa.infn.it
}

\begin{abstract}
By resorting to some results in quantum field theories with spontaneous breakdown of symmetry we show that an explanation based on microscopic dynamics can be given of the fact that topological defect formation is observed during the process of non-equilibrium phase transitions characterized by a non-zero order parameter. We show that the NambuGoldstone particle acquires an effective non-zero mass due to boundary (finite volume) effects and this is related with the size of the defect. We also relate such volume effects with temperature effects.
\end{abstract}

PACS: 03.70.+k, 11.30.Qc, 95.50.Eb

Much attention is currently devoted to the problem of topological defect formation during the process of symmetry breaking phase transitions [1]. In such a process it may happen that a region, surrounded by ordered domains, remains trapped in the "normal" or symmetric state. This occurrence manifests as a topological defect. Topological defects are observed as macroscopic extended objects with classical behavior, e.g. vortices in superconductors and superfluids, magnetic domain walls in ferromagnets, dislocations, grain boundaries, point defects in crystals. In cosmology, topological defects, such as cosmic strings, may have been playing a rôle in the phase transition processes in the early Universe [2]. The Kibble-Zurek scenario 3, 4] provides the phenomenological understanding of the defect formation in phase transitions. There is a surprising analogy between defect formation in solid state physics and in high energy physics and cosmology [5]. For an interesting table of analogies see [6]. As an example, we just mention the analogy between vortex in superfluids and global cosmic strings. Studying the physics of defect formation in condensed matter physics may be then helpful in the understanding of possible scenarios in the early Universe cosmology [1]. The 
analysis of the formation of defects in phase transitions thus becomes a "diagnostic tool" [7] in the study of non-equilibrium symmetry breaking processes in a wide range of energy scale.

In this paper, by limiting ourselves to the class of non-equilibrium symmetry breaking phase transitions characterized by an order parameter, we discuss some of the questions which arise in the study of defect formation. For example, which one is the connection between defect formation and symmetry breaking, i.e., more precisely, why are extended objects with topological singularity observed only in systems showing some sort of ordered patterns? Why defect formation is observed during the processes of phase transitions? Why are the features of the defect formation so general, i.e. why are they shared by quite different systems, from condensed matter to cosmology? How to explain in a unified theoretical scheme the macroscopic behavior of extended objects and their interaction with quanta? How temperature and finite volume (the system boundaries) effects fit in such a theoretical frame?

Of course, there is a large body of literature in quantum field theory (QFT) on the dynamics of defect formation and some of the above questions have been, at least partially, answered. By resorting indeed to some of such works, in the present paper we reach the following main results:

i) we show that a theoretical explanation based on microscopic dynamics can be given of the fact that topological defect formation is observed during the process of non-equilibrium symmetry breaking phase transitions (limited to the case of the existence of the order parameter and in the presence of a gauge field).

ii) we provide an explicit proof that the Nambu-Goldstone (NG) particle acquires an effective non-zero mass due to boundary (finite volume) effects, which is related with the size of the defect. We also relate such volume effects with temperature effects.

Our derivation is based on symmetry properties of the dynamics at the basic level of the quantum fields. As far as we know, in the literature the conclusions we reach are commonly accepted mostly on the basis of phenomenological arguments. Also, our conclusions are model independent, which may explain why some of the features of defect formation, within the limitations imposed to our analysis, are shared by many systems in a wide range of energy scale, independently of specific aspects of the system dynamics. Moreover, our results apply to the case of the Abelian U(1) symmetry (for vortices) as well as to the non-Abelian case of $\mathrm{SO}(3)$ and $\mathrm{SU}(2)$ symmetry (as for the monopole and the sphaleron case, respectively). As already mentioned, throughout this paper our discussion is limited to the case in which an order parameter exists and a gauge field is present. However, we also present some comments on boundary and temperature effects on the kink defect.

We arrive at the result i) as a direct consequence of two known properties of gauge theories with spontaneous breakdown of symmetry (SBS). Both these properties have to do with the topological characterization of the non-homogeneous boson condensation in SBS theories. Although these properties are known since several years, nevertheless they have not been considered till now in relation with the problem of the appearance of topological defects in phase transitions. For a preliminary discussion see ref. [8]. For the reader convenience we report in the Appendices some details of the mathematical formalism to which we have to resort in our discussion.

Let us start by recalling that in QFT the formation of extended objects is described by non-homogeneous boson condensation [9] - [12]. This condensation is formally obtained by translations of boson fields (not necessarily massless), say $\chi_{i n}(x) \rightarrow \chi_{i n}(x)+f(x)$, with c- 
number function $f(x)$, satisfying the same field equation for $\chi_{i n}(x)$. These translations are called boson transformations [9]. The boson transformation function plays the rôle of a "form factor" and the extended object appears as the macroscopic envelop of the non-homogeneous boson condensate (localized over a finite domain). The topological charge of the extended object (the defect) arises from the topological singularity of the boson condensation function.

We remark that, on the other hand, transitions between the system phases characterized by different ordered patterns in the ground state are induced by variations (gradients) of the NG boson condensate, i.e. of boson transformation function. Hence, in order to show that in such a process the conditions are met for the formation of topological defects, we need

(a) to consider under which constraints the boson transformation function $f(x)$ can carry a topological singularity and

(b) to show that in the process of phase transitions under study these constraints are in fact satisfied.

Let us first consider the point (a). We recall that in theories where boson condensation can occur, topological defects are observed only in systems exhibiting ordered patterns (characterized by a non-zero order parameter).

In fact one can show that boson transformation functions carrying topological singularities are allowed only for massless bosons [9, 10], such as NG bosons of SBS theories where ordered ground states appear. The proof of this goes as follows [10].

Consider the boson transformation $\chi_{i n}(x) \rightarrow \chi_{i n}(x)+f(x)$. Let $f(x)$ carry a topological singularity, which means that it is path-dependent:

$$
G_{\mu \nu}^{\dagger}(x) \equiv\left[\partial_{\mu}, \partial_{\nu}\right] f(x) \neq 0, \quad \text { for certain } \mu, \nu, x .
$$

We will see below that $\partial_{\mu} f$ is related with observables and therefore it is single-valued, i.e. $\left[\partial_{\rho}, \partial_{\nu}\right] \partial_{\mu} f(x)=0$. Recall that $f(x)$ is solution of the $\chi_{i n}$ equation and suppose there is a non-zero mass term: $\left(\partial^{2}+m^{2}\right) f(x)=0$. From the regularity of $\partial_{\mu} f(x)$ it follows that

$$
\partial_{\mu} f(x)=\frac{1}{\partial^{2}+m^{2}} \partial^{\lambda} G_{\lambda \mu}^{\dagger}(x),
$$

which leads to $\partial^{2} f(x)=0$, which in turn implies $m=0$. Thus (1) is compatible only with massless $\chi_{i n}$. This explains why topological defects are observed only in systems exhibiting ordered patterns, namely in the presence of NG boson condensation sustaining the long range ordering correlation.

We remark that the above conclusion is not limited by dimensional considerations or by the Abelian or the non-Abelian nature of the theory symmetry group. It can be easily extended to the non-relativistic regime (see [10]) and it holds true in a theory with or without gauge fields. It applies to a full set of topologically non-trivial extended objects, ranging from topological line singularity, to surface singularity, point defects, grain boundaries and dislocation defects in crystals, SU(2)-triplet model and monopole singularity. The topological singularity and the topological charge of the related extended object can be completely characterized.For a detailed account see ref. [10], especially Chapter 10.

Next, for the point (b), we consider the case in which a gauge field is present. We then observe that from Eq. (28) (Appendix B) we obtain the classical ground state current $j_{\mu}$ as

$$
j_{\mu}(x) \equiv\left\langle 0\left|j_{H \mu}(x)\right| 0\right\rangle=m_{V}^{2}\left[a_{\mu}(x)-\frac{1}{e_{0}} \partial_{\mu} f(x)\right],
$$


where $m_{V}^{2} a_{\mu}(x)$ is the Meissner-like current and $\frac{m_{V}^{2}}{e_{0}} \partial_{\mu} f(x)$ is the boson current.

Note that the observable classical current is given in terms of gradients $\partial_{\mu} f$ of the boson condensation function. Eq. (3) shows that the macroscopic ground state effects, such as the classical field and the classical current, do not occur for regular $f(x)\left(G_{\mu \nu}^{\dagger}=0\right)$. In fact, from (28) we obtain $\partial^{2} a_{\mu}(x)=0$ for regular $f(x)$, i.e. $a_{\mu}(x)=\frac{1}{e_{0}} \partial_{\mu} f(x)$, which in turn implies zero classical current $\left(j_{\mu}=0\right)$ and zero classical field $\left(F_{\mu \nu}=\partial_{\mu} a_{\nu}-\partial_{\nu} a_{\mu}\right)$ (the Meissner-like current and the boson current cancel each other).

The conclusion is that the vacuum current appears only when $f(x)$ has topological singularities, which, as we have seen above (point (a)), is only compatible with the condensation of massless bosons, i.e. when SBS occurs. Again this conclusion is a general one, it does not depend on the specific model. It holds for Abelian as well as for non-Abelian groups, in the relativistic and in the non-relativistic case.

Summing up, in a gauge theory, the symmetry breaking phase transitions characterized by macroscopic ground state effects, such as the vacuum current and field, (e.g. in superconductors) can occur only when there are non-zero gradients of topologically non trivial condensation of $N G$ bosons. But this means that the conditions for the formation of topological defects are met and thus we see why topological defects are observed in the process of symmetry breaking phase transitions. We have thus obtained our result i).

Notice that the appearance of space-time dependent order parameter $\tilde{v}$ is no guarantee that persistent ground state currents (and fields) will exist: if $f$ is a regular function, the space-time dependence of $\tilde{v}$ can be gauged away by an appropriate gauge transformation. Which means that non-homogeneous condensation, and thus extended objects, cannot be obtained in such case. Also note that in a theory which has only global gauge invariance non-singular boson transformations of the NG fields can produce non-trivial physical effects (like linear flow in superfluidity).

In conclusion, in a gauge theory with non-zero order parameter, in order to have topological defects one needs boson condensation function with topological singularities and this is only allowed for NG boson condensation. These conditions are all met in the process of phase transition in such a theory.

A further consequence of the above analysis is that, since the boson transformation with regular $f$ does not affect observable quantities, the $S$ matrix must be actually given by (cf. Appendix A)

$$
S=: S\left[\rho_{i n}, U_{i n}^{\mu}-\frac{1}{m_{V}} \partial\left(\chi_{i n}-b_{i n}\right)\right]: .
$$

This is in fact independent of the boson transformation with regular $f$ :

$$
S \rightarrow S^{\prime}=: S\left[\rho_{i n}, U_{i n}^{\mu}-\frac{1}{m_{V}} \partial\left(\chi_{i n}-b_{i n}\right)+Z_{3}^{-\frac{1}{2}}\left(a^{\mu}-\frac{1}{e_{0}} \partial^{\mu} f\right)\right]:
$$

since $a_{\mu}(x)=\frac{1}{e_{0}} \partial_{\mu} f(x)$ for regular $f$. However, $S^{\prime} \neq S$ for singular $f$ : in such a case (可) shows that $S^{\prime}$ includes the interaction of the quanta $U_{i n}^{\mu}$ and $\rho_{i n}$ with the classical field and current. This shows how it may happens that quanta interact and have effects on classically behaving macroscopic extended objects.

For the point ii), let us consider now the effects of finite volume on homogeneous boson condensation.

For large but finite volume we expect that the symmetry breakdown order parameter is constant "inside the bulk" far from the boundaries. However, "near" the boundaries, one 
might expect "distortions" in the order parameter: "near" the system boundaries we may have non-homogeneous order parameter, $\tilde{v}=\tilde{v}(x)$ (or even $\tilde{v} \rightarrow 0$ ). Such non-homogeneities in the boson condensation will "smooth out" in the $V \rightarrow \infty$ limit.

From the Ward-Takahashi identities one obtains the following pole structure for the twopoint function of the $\chi(x)$ field [13]:

$$
\langle\chi(x) \chi(y)\rangle=\lim _{\epsilon \rightarrow 0}\left\{\frac{i}{(2 \pi)^{4}} \int d^{4} p \frac{Z_{\chi} e^{-i p(x-y)}}{p^{2}-m_{\chi}^{2}+i \epsilon a_{\chi}}+(\text { continuum contributions })\right] .
$$

$Z_{\chi}$ and $a_{\chi}$ are renormalization constants. The space integration of $\langle\chi(x) \chi(y)\rangle$ then picks up the pole contribution at $p^{2}=0$, and leads to [14, 15]

$$
\tilde{v}=\frac{Z_{\chi}}{a_{\chi}} v \Leftrightarrow m_{\chi}=0, \quad \text { or } \quad \tilde{v}=0 \Leftrightarrow m_{\chi} \neq 0
$$

where $v$ denotes a convenient c-number [13]. Eq. (7) expresses the well known Goldstone theorem: if the symmetry is spontaneously broken $(\tilde{v} \neq 0)$, the NG massless mode exists, whose interpolating Heisenberg field is $\chi_{H}(x)$. Since it is massless it manifests as a long range correlation mode and it is thus responsible for the vacuum ordering, as already observed above.

Restrict now the space integration of Eq. (6) over the finite (but large) volume $V \equiv \eta^{-3}$ and use for each space component of $p$ :

$$
\delta_{\eta}(p)=\frac{1}{2 \pi} \int_{-\frac{1}{\eta}}^{\frac{1}{\eta}} d x e^{i p x}=\frac{1}{\pi p} \sin \frac{p}{\eta}
$$

which, as well known, approaches $\delta(p)$ as $\eta \rightarrow 0: \lim _{\eta \rightarrow 0} \delta_{\eta}(p)=\delta(p)$. Consider that

$$
\lim _{\eta \rightarrow 0} \int d p \delta_{\eta}(p) f(p)=f(0)=\lim _{\eta \rightarrow 0} \int d p \delta(p-\eta) f(p) .
$$

Then, using $\delta_{\eta}(p) \simeq \delta(p-\eta)$ for small $\eta$, one obtains

$$
\tilde{v}(y, \epsilon, \eta)=i \epsilon v e^{-i \eta \cdot \mathbf{y}} \Delta_{\chi}\left(\epsilon, \eta, p_{0}=0\right),
$$

with

$$
\Delta_{\chi}\left(\epsilon, \eta, p_{0}=0\right)=\left[\frac{Z_{\chi}}{-\omega_{\mathbf{p}=\eta}^{2}+i \epsilon a_{\chi}}+(\text { continuum contributions })\right],
$$

and $\omega^{2} \mathbf{p = \eta}=\eta^{2}+m_{\chi}{ }^{2}$. Thus, $\lim _{\epsilon \rightarrow 0} \lim _{\eta \rightarrow 0} \tilde{v}(y, \epsilon, \eta) \neq 0$ only if $m_{\chi}=0$, otherwise $\tilde{v}=0$. Note that the Goldstone theorem is thus recovered in the infinite volume limit $(\eta \rightarrow 0)$.

On the other hand, if $m_{\chi}=0$ and $\eta$ is given a non-zero value (i.e. by reducing to a finite volume, i.e. in the presence of boundaries), then $\omega_{\mathbf{p}=\eta} \neq 0$ and it acts as an "effective mass" for the $\chi$ bosons. Then, in order to have the order parameter $\tilde{v}$ different from zero $\epsilon$ must be kept non-zero.

In conclusion, near the boundaries $(\eta \neq 0)$ the NG bosons acquire an effective mass $m_{e f f} \equiv \omega_{\mathbf{p}=\eta}$. They will then propagate over a range of the order of $\xi \equiv \frac{1}{\eta}$, which is the linear size of the condensation domain, or, in the presence of topological singularity, the size of the topologically non-trivial condensation, namely of the extended object (the defect). 
We stress that if $\eta \neq 0$ then $\epsilon$ must be non-zero in order to have the order parameter different from zero: $\tilde{v} \neq 0$ (at least locally). In such a case the symmetry breakdown is maintained because $\epsilon \neq 0$ : $\epsilon$ acts as the coupling with an external field (the pump) providing energy. Energy supply is required in order to condensate modes of non-zero lowest energy $\omega_{\mathbf{p}=\eta}$. In summary, boundary effects are in competition with the breakdown of symmetry [8]. They may preclude its occurrence or, if symmetry is already broken, they may reduce to zero the order parameter.

Temperature may have similar effects on the order parameter (at $T_{C}$ symmetry may be restored). Since the order parameter goes to zero when NG modes acquire non-zero effective mass (unless, as seen, external energy is supplied), we may then represent the effect of thermalization in terms of finite volume effects and put, e.g., $\eta \propto \sqrt{\frac{\left|T-T_{C}\right|}{T_{C}}}$. In this way temperature fluctuations around $T_{C}$ may produce fluctuations in the size $\xi$ of the condensed domain (the size of ordered domain and/or of the domain where non-homogeneous condensation occurs, namely of the defect).

For example, suppose to start with $T>T_{C}$, but near to $T_{C}$. In the presence of an external driving field $(\epsilon \neq 0)$, even before transition to fully ordered phase is achieved (as $T \rightarrow T_{C}$ ), one may have the formation of ordered domains of size $\xi \propto\left(\sqrt{\frac{\left|T-T_{C}\right|}{T_{C}}}\right)^{-1}$. As far as $\eta \neq 0$, the ordered domains (and the topological defects) are unstable, they disappear as the external field coupling $\epsilon \rightarrow 0$.

Of course, assuming $T$ is lowered below $T_{C}$, if ordered domains are still present at $T<T_{C}$, they also disappear as $\epsilon \rightarrow 0$. The possibility to maintain such ordered domains below $T_{C}$ depends on the speed at which $T$ is lowered, compared to the speed at which the system is able to get homogeneously ordered. Notice that the speed at which $T \rightarrow T_{C}$ is related to the speed at which $\eta \rightarrow 0$.

In the case of the kink solution, the boson transformation function $f_{\beta}(x)=$ const. $e^{-\mu_{0}(\beta) x_{1}}$ plays the role of "form factor" for the soliton solution. The number of condensed bosons is proportional to $\left|f_{\beta}(x)\right|^{2}=e^{-2 \mu_{0}(\beta)\left(x_{1}-a\right)}$, which is maximal near the kink center $x_{1}=a$ and decreases over a size $\xi_{\beta}=\frac{2}{\mu_{0}(\beta)}$. The mass $\mu_{0}=(2 \lambda)^{\frac{1}{2}} v(\beta)$, with [11] $v^{2}(\beta)=\tilde{v}^{2}-3\left\langle: \rho^{2}\right.$ : \rangle$_{0}<\tilde{v}^{2}$, of the "constituent" fields $\rho^{i n}(x)$ fixes the kink size $\xi_{\beta} \propto \frac{2}{\mu_{0}}=\frac{\sqrt{2}}{\sqrt{\lambda} v(\beta)}$, which thus increases as $T$ increases: $T \rightarrow T_{C}$ (say $T \neq T_{C}$ but near $T_{C}$. At $T_{C}, v\left(\beta_{C}\right)=0$.).

In the $T \rightarrow 0$ limit the kink size is $\xi_{0} \propto \frac{\sqrt{2}}{\sqrt{\lambda} \tilde{v}}<\frac{\sqrt{2}}{\sqrt{\lambda} v(\beta)}=\xi_{\beta}$. For $T$ different from zero, the thermal Bose condensate $\left\langle: \rho^{2}:\right\rangle_{0}$ develops, which acts as a potential term for the kink field. It is such a potential term which actually controls the "size" (and the number) of the kinks. Only far from the kink core, namely in the limit $v(x, \beta) \rightarrow$ const, the $\rho^{\text {in }}(x)$ field may be considered as a free field. The translation of the boson field by $f_{\beta}$ breaks the homogeneity of the otherwise constant in space order parameter $v(\beta)$.

Let us close by observing that in the case of topologically non-trivial condensation at finite temperature the order parameter $v(x, \beta)$ provides a mapping between the variation domains of $(x, \beta)$ and the space of the unitarily inequivalent representations of the canonical commutation relations, i.e. the set of Hilbert spaces where the operator fields are realized for different values of the order parameter. This expresses the known fact that we have nontrivial homotopy mappings between the $(x, \beta)$ variability domain and the group manifold. As well known, in the vortex case one has the mapping $\pi$ of $S^{1}$, surrounding the $r=0$ singularity, to the group manifold of $\mathrm{U}(1)$ which is topologically characterized by the winding number 
$n \in Z \in \pi_{1}\left(S^{1}\right)$. It is such a singularity which is carried by the boson condensation function of the NG modes. In the monopole case [11], the mapping $\pi$ is the one of the sphere $S^{2}$, surrounding the singularity $r=0$, to $\mathrm{SO}(3) / \mathrm{SO}(2)$ group manifold, with homotopy classes of $\pi_{2}\left(S^{2}\right)=Z$. Same situation occurs in the sphaleron case [1], provided one replaces $\mathrm{SO}(3)$ and $\mathrm{SO}(2)$ with $\mathrm{SU}(2)$ and $\mathrm{U}(1)$, respectively.

In conclusion, transitions between phases characterized by an order parameter imply "moving" over unitarily inequivalent representations, and this is achieved by gradients in NG boson condensation function. In the presence of a gauge field, macroscopic ground state field and currents can only be obtained by non-homogeneous NG boson condensation with topological singularities. In turn, the occurrence of such topologically non-trivial condensation allows the formation of topological defects: this explains why topological defect formation is observed in such a kind of symmetry breaking phase transition processes ("where the defects come from").

We have also seen that finite volume (the presence of boundaries) is related to the size of the ordered domains and of the defects and we have briefly discussed the correlation with temperature effects. This may justify the expectation relating the number of observed defects with the non-equilibrium dynamics of phase transitions.

In the case of the kink there are no NG modes, nevertheless the topologically non-trivial kink solution requires the boson condensation function to carry divergence singularity (at spatial infinity).

This work has been partially supported by INFN, INFM, MURST and the ESF Network on Topological defect formation in phase transitions. 
In the standard Lehmann-Symanzik-Zimmermann (LSZ) formalism [16] of QFT, the dynamics is given in terms of the interacting fields, say $\varphi_{H}(x)$, also called the Heisenberg fields. On the other hand, observables are described by asymptotic in- (or out-) operator fields (physical fields), say $\varphi_{i n}(x)$, which satisfy free field equations. The mechanisms described in this appendix are fully general, however, for simplicity we consider the Lagrangian density $\mathcal{L}\left[\phi_{H}(x), \phi_{H}{ }^{*}(x), A_{H \mu}(x)\right]$ for a complex scalar field $\phi_{H}(x)$ interacting with a gauge field $A_{H \mu}(x)$. We do not need to specify the detailed structure of the Lagrangian. We only require that such a Lagrangian be invariant under global and local U(1) gauge transformations (as, e.g., in the Higgs-Kibble model [17, 18]):

$$
\begin{array}{ccc}
\phi_{H}(x) \rightarrow e^{i \theta} \phi_{H}(x) & , & A_{H \mu}(x) \rightarrow A_{H \mu}(x), \\
\phi_{H}(x) \rightarrow e^{i e_{0} \lambda(x)} \phi_{H}(x) & , & A_{H \mu}(x) \rightarrow A_{H \mu}(x)+\partial_{\mu} \lambda(x),
\end{array}
$$

respectively, with $\lambda(x) \rightarrow 0$ for $\left|x_{0}\right| \rightarrow \infty$ and/or $|\mathbf{x}| \rightarrow \infty$. We use the Lorentz gauge $\partial^{\mu} A_{H \mu}(x)=0$ and put $\phi_{H}(x)=\frac{1}{\sqrt{2}}\left[\psi_{H}(x)+i \chi_{H}(x)\right]$. We also assume that SBS can occur: $\left\langle 0\left|\phi_{H}(x)\right| 0\right\rangle \equiv \tilde{v} \neq 0$, with $\tilde{v}$ constant and put $\rho_{H}(x) \equiv \psi_{H}(x)-\tilde{v}$. It is known [13] that the theory contains a massless negative norm field (ghost) $b_{i n}(x)$, the Nambu-Goldstone massless mode $\chi_{i n}(x)$, and a massive vector field $U_{i n}^{\mu}$. The relation between the dynamics and the observable properties of the physical states is provided by the LSZ mapping (the dynamical map, or Haag expansion) between interacting fields and physical fields. In the present case one finds [13] the following LSZ maps:

$$
\begin{gathered}
\phi_{H}(x)=: \exp \left\{i \frac{Z_{\chi}^{\frac{1}{2}}}{\tilde{v}} \chi_{i n}(x)\right\}\left[\tilde{v}+Z_{\rho}^{\frac{1}{2}} \rho_{i n}(x)+F\left[\rho_{i n}, U_{i n}^{\mu}, \partial\left(\chi_{i n}-b_{i n}\right)\right]\right]: \\
A_{H}^{\mu}(x)=Z_{3}^{\frac{1}{2}} U_{i n}^{\mu}(x)+\frac{Z_{\chi}^{\frac{1}{2}}}{e_{0} \tilde{v}} \partial^{\mu} b_{i n}(x)+: F^{\mu}\left[\rho_{i n}, U_{i n}^{\mu}, \partial\left(\chi_{i n}-b_{i n}\right)\right]: .
\end{gathered}
$$

$Z_{\chi}, Z_{\rho}$ and $Z_{3}$ are the wave function renormalization constants. As usual, the colon symbol denotes normal ordering. The functionals $F$ and $F^{\mu}$ have to be determined within a specific choice for the Lagrangian. The $S$-matrix is given by $S=: S\left[\rho_{i n}, U_{i n}^{\mu}, \partial\left(\chi_{i n}-b_{i n}\right)\right]:$ It is important to notice that the dynamical mappings (14) and (15) (and the one for the $S$ matrix) are weak equalities, i.e. they are equalities among matrix elements computed in the Fock space for the physical states. The free field equations are

$$
\begin{gathered}
\partial^{2} \chi_{i n}(x)=0, \quad \partial^{2} b_{i n}(x)=0, \quad\left(\partial^{2}+m_{\rho}^{2}\right) \rho_{i n}(x)=0, \\
\left(\partial^{2}+m_{V}^{2}\right) U_{i n}^{\mu}(x)=0 \quad, \quad \partial_{\mu} U_{i n}^{\mu}(x)=0 .
\end{gathered}
$$

with $m_{V}^{2}=\frac{Z_{3}}{Z_{\chi}}\left(e_{0} \tilde{v}\right)^{2}$.

One can show that the local gauge transformations and the global phase transformations (cf. (13) and (12) ) of the Heisenberg fields are induced by the in-field transformations (see (14) and (15)):

$$
\chi_{i n}(x) \rightarrow \chi_{i n}(x)+\frac{e_{0} \tilde{v}}{Z_{\chi}^{\frac{1}{2}}} \lambda(x), \quad b_{i n}(x) \rightarrow b_{i n}(x)+\frac{e_{0} \tilde{v}}{Z_{\chi}^{\frac{1}{2}}} \lambda(x),
$$




$$
\rho_{i n}(x) \rightarrow \rho_{i n}(x), \quad U_{i n}^{\mu}(x) \rightarrow U_{i n}^{\mu}(x)
$$

and by

$$
\begin{gathered}
\chi_{i n}(x) \rightarrow \chi_{i n}(x)+\frac{\tilde{v}}{Z_{\chi}^{\frac{1}{2}}} \theta f(x), \\
b_{i n}(x) \rightarrow b_{i n}(x) \quad, \rho_{i n}(x) \rightarrow \rho_{i n}(x) \quad, U_{i n}^{\mu}(x) \rightarrow U_{i n}^{\mu}(x),
\end{gathered}
$$

respectively. The square integrable function $f(x)$ is required to be solution of $\partial^{2} f(x)=$ 0 . Note that (20) with $f(x)=1$ (translation by a constant c-number) is not unitarily implementable. $f(x)$ is introduced in order to make the generator of such a transformation well defined. The limit $f(x) \rightarrow 1$ (i.e. the infinite volume limit) is to be performed at the end of the computation. Notice that the in-field equations and the $S$ matrix are invariant under the above in-field transformations (in the limit $f \rightarrow 1$ ).

The transformation (20) with $f(x)=1$ describes the homogeneous boson condensation. Once NG bosons are condensed in the ground state, long range correlation is established which manifests as the ordered pattern in the system ground state. This means that transitions between the system phases characterized by different ordered patterns in the ground state may be induced by the process of boson condensation. In other words, phase transitions (transitions among unitarily inequivalent Fock spaces) are induced by variations (gradients) of the NG boson condensate.

The fact that the local and the global U(1) gauge transformations are induced by the group $G^{\prime}$ of transformations (18-21) is named the dynamical rearrangement of symmetry [9, 10]: The dynamical rearrangement of symmetry expresses the consistency between the invariance of the Lagrangian under the symmetry transformations and the SBS condition $\left\langle 0\left|\phi_{H}(x)\right| 0\right\rangle=\tilde{v} \neq 0$.

Translations of boson fields (eqs. (18) and (20)) are thus obtained as a consequence of SBS. As a general result one finds that $G^{\prime}$ is the group contraction of the symmetry group of the dynamics $(\mathrm{U}(1)$ in our present case; more generally, if the symmetry group is $\mathrm{SU}(\mathrm{n})$ or $\mathrm{SO}(\mathrm{n})$, the group contraction $G^{\prime}$ is $\mathrm{EU}(\mathrm{n}-1)$ or $\mathrm{E}(\mathrm{n})$, respectively) [19. Notice that $G^{\prime}$ is the transformation group relevant to the phase transitions process.

Appendix B. Macroscopic field and currents generated by the boson condensation

In the framework of the model in Appendix A, the Maxwell equations are

$$
-\partial^{2} A_{H}^{\mu}(x)=j_{H}^{\mu}(x)-\partial^{\mu} B(x)
$$

with

$$
B(x)=\frac{e_{0} \tilde{v}}{Z_{\chi}^{\frac{1}{2}}}\left[b_{i n}(x)-\chi_{i n}(x)\right], \quad \partial^{2} B(x)=0,
$$

and with $j_{H}^{\mu}(x)=\delta \mathcal{L}(x) / \delta A_{H \mu}(x)$. We may require that the current $j_{H}^{\mu}$ is the only source of the gauge field $A_{H}^{\mu}$ in any observable process. This is obtained by imposing the condition: ${ }_{p}\left\langle b\left|\partial^{\mu} B(x)\right| a\right\rangle_{p}=0$, i.e.

$$
\left(-\partial^{2}\right)_{p}\left\langle b\left|A_{H}^{0 \mu}(x)\right| a\right\rangle_{p}={ }_{p}\left\langle b\left|j_{H}^{\mu}(x)\right| a\right\rangle_{p},
$$


where we use $A_{H}^{0 \mu}(x) \equiv A_{H}^{\mu}(x)-e_{0} \tilde{v}: \partial^{\mu} b_{i n}(x): .|a\rangle_{p}$ and $|b\rangle_{p}$ denote two generic physical states. The condition ${ }_{p}\left\langle b\left|\partial^{\mu} B(x)\right| a\right\rangle_{p}=0$ leads to the Gupta-Bleuler-like condition

$$
\left[\chi_{i n}^{(-)}(x)-b_{i n}^{(-)}(x)\right]|a\rangle_{p}=0,
$$

with $\chi_{i n}^{(-)}$and $b_{i n}^{(-)}$the positive-frequency parts of the corresponding fields. $\chi_{i n}$ and $b_{i n}$ do not participate to any observable reaction. However, we stress that the NG bosons do not disappear from the theory: their condensation in the vacuum can have observable effects. Notice that Eq.(24) are the classical Maxwell equations.

We now remark that the boson transformation must be also compatible with the physical state condition (25). Under the boson transformation $\chi_{i n}(x) \rightarrow \chi_{i n}(x)+\frac{\tilde{v}}{Z_{\chi}^{\frac{1}{2}}} f(x), B$ changes as

$$
B(x) \rightarrow B(x)-\frac{e_{0} \tilde{v}^{2}}{Z_{\chi}} f(x) .
$$

Eq. (24) is then violated. In order to restore it, the shift in $B$ must be compensated by means of the transformation on $U_{i n}$ :

$$
U_{i n}^{\mu}(x) \rightarrow U_{i n}^{\mu}(x)+Z_{3}{ }^{-\frac{1}{2}} a^{\mu}(x) \quad, \quad \partial_{\mu} a^{\mu}(x)=0,
$$

with a convenient c-number function $a^{\mu}(x)$. The dynamical maps of the various Heisenberg operators are not affected by (27) provided

$$
\left(\partial^{2}+m_{V}^{2}\right) a_{\mu}(x)=\frac{m_{V}^{2}}{e_{0}} \partial_{\mu} f(x) .
$$

This is the Maxwell equation for the vector potential $a_{\mu}$ [13, 12]. A similar result may be obtained in more complex models with non-Abelian symmetry groups [10, 11]. 


\section{Bibliography}

[1] Y.M. Bunkov and H. Godfrin (Eds.), Topological defects and the non-equilibrium dynamics of symmetry breaking phase transitions, NATO Science Series C 549, (Kluwer Acad. Publ. Dordrecht 2000).

[2] T.W.B. Kibble, J. Phys. A9, 1387 (1976); Phys. Rep. 67, 183 (1980). A. Vilenkin, Phys. Rep. 121, 264 (1985).

[3] T.W.B. Kibble, in Topological defects and the non-equilibrium dynamics of symmetry breaking phase transitions, eds. Y.M. Bunkov and H. Godfrin, NATO Science Series C 549, (Kluwer Acad. Publ. Dordrecht 2000), p. 7.

[4] W.H. Zurek, Phys. Rep. 276, 177 (1997) and refs. therein quoted

[5] G.E. Volovik, in Topological defects and the non-equilibrium dynamics of symmetry breaking phase transitions, eds. Y.M. Bunkov and H. Godfrin, NATO Science Series C 549, (Kluwer Acad. Publ. Dordrecht 2000), p. 353.

[6] G.E. Volovik, in Topological defects and the non-equilibrium dynamics of symmetry breaking phase transitions, eds. Y.M. Bunkov and H. Godfrin, NATO Science Series C 549, (Kluwer Acad. Publ. Dordrecht 2000), p. 1.

[7] W.H. Zurek, L.M.A.Bettencourt, J.Dziarmaga and N.D.Antunes, in Topological defects and the non-equilibrium dynamics of symmetry breaking phase transitions, eds. Y.M. Bunkov and H. Godfrin, NATO Science Series C 549, (Kluwer Acad. Publ. Dordrecht 2000), p. 77.

[8] G. Vitiello, in Topological defects and the non-equilibrium dynamics of symmetry breaking phase transitions, eds. Y.M. Bunkov and H. Godfrin, NATO Science Series C 549, (Kluwer Acad. Publ. Dordrecht 2000), p.171.

[9] H. Umezawa, Advanced Field Theory: Micro, Macro and Thermal Physics (American Institute of Physics, 1993).

[10] H. Umezawa, H. Matsumoto, and M. Tachiki, Thermo Field Dynamics and Condensed States, (North-Holland Publ.Co., Amsterdam, 1982).

[11] R. Manka and G. Vitiello, Ann. Phys. (N.Y.) 199, 61 (1990).

[12] H. Matsumoto, N.J. Papastamatiou, and H. Umezawa, Nucl. Phys. B97, 90 (1975). 
[13] H. Matsumoto, N.J. Papastamatiou, H. Umezawa and G. Vitiello, Nucl. Phys. B97, 61 (1975).

[14] H. Matsumoto, N.J. Papastamatiou and H. Umezawa, Nucl. Phys. B68, 236 (1974).

[15] H. Matsumoto, N.J. Papastamatiou and H. Umezawa, Nucl. Phys. B82, 45 (1974).

[16] C. Itzykson and J.B. Zuber, Quantum Field Theory (MacGraw-Hill Book Co., N.Y. 1980)

[17] P. Higgs, Phys. Rev. 45, 1156 (1960).

[18] T.W.B. Kibble, Phys. Rev. 155, 1554 (1967).

[19] C. De Concini and G. Vitiello, Nucl. Phys. B116, 141 (1976). 